\title{
Time-reparametrization invariance in eternal inflation
}

\author{
Sergei Winitzki \\ Department of Physics, Ludwig-Maximilians University, Theresienstr. 37, 80333 Munich, Germany
}

\begin{abstract}
I address some recently raised issues regarding the time-parametrization dependence in stochastic descriptions of eternal inflation. To clarify the role of the choice of the time gauge, I show examples of gauge-dependent as well as gauge-independent statements about physical observables in eternally inflating spacetimes. In particular, the relative abundance of thermalized and inflating regions is highly gauge-dependent. The unbounded growth of the 3-volume of the inflating regions is found in certain time gauges, such as the proper time or the scale factor gauge. Yet in the same spacetimes there exist time foliations with a finite and monotonically decreasing 3-volume, which I demonstrate by an explicit construction. I also show that there exists no "correct" choice of the time gauge that would yield an unbiased stationary probability distribution for observables in thermalized regions.
\end{abstract}

\section{INTRODUCTION}

In most inflationary scenarios, the exit from inflation and the subsequent reheating do not occur everywhere at once. Some regions of the universe finish inflation and thermalize, while other regions continue inflating. Generic models of inflation predict that such inflating regions will be found at arbitrarily late times. This phenomenon was called eternal inflation ${ }^{1}$ [1, 2, 3]. The inflating regions expand much faster than the thermalized regions, so heuristically one expects that most of the volume of the universe is dominated by regions that have been inflating for a very long time and thus retain no memory of the initial state. The planet Earth is equally likely to be in any of the (infinitely many) thermalized regions, hence our present-day observations are essentially independent of the conditions before the onset of inflation. In this way the picture of eternal inflation was found to alleviate the problem of initial conditions for inflation. Any initial state is permissible (agrees with our present observations) as long as there is a nonzero probability for eternal inflation to set in.

The inflating (i.e. not yet thermalized) domain in an eternally inflating spacetime can be described as a random collection of horizon-sized regions with locally de Sitter geometry. All regions expand at different local Hubble rates $H(t, x)$, where $x$ is a comoving coordinate and $t$ is the proper time measured along comoving geodesics $x=$ const. A region with expansion rate $H$ evolves during one Hubble time $\Delta t \sim H^{-1}$ into $e^{3}$ new horizon-sized regions having slightly changed expansion rates; some of these new regions may accidentally thermalize. Thus the evolution of the spacetime can be viewed as a realization of a branching diffusion process. One can compute probability distributions for various physical observables, such as the scalar field $\phi$ or the scale factor $a$, at a given time $t$. Calculations are performed by modeling the branching diffusion process using Langevin

\footnotetext{
1 Since it was not implied that inflation is eternal also to the past,
} a more precise designation would be "future-eternal inflation." and Fokker-Planck (FP) equations (see [4] for a review and further references). Below I shall summarily refer to this description as the FP formalism.

A description through an equal-time probability distribution necessarily suffers from a dependence on the choice of the time variable $t$ and in some cases this gauge dependence is quite dramatic [4, 5, 6]. The source of the gauge dependence is a bias introduced into the selection of observers when a particular equal-time surface is chosen. For example, the proper-time gauge favors recently thermalized regions, as compared with the scalefactor gauge. Probability distributions can be defined in a gauge-invariant manner in some cases, for instance in a class of models where an observable assumes all possible values within one causally connected thermalized patch [7, 8]. However, a straightforward gauge-invariant definition of probability distributions is still lacking in the important case where observables assume different values in causally disconnected patches (for some approaches, see $[9]$ ).

In this paper I shall not consider these gauge-invariant prescriptions but instead focus on the issues of gauge dependence in the usual FP formalism. This apparently unavoidable gauge dependence has caused some consternation [10,11] regarding the overall validity of the standard picture of eternal inflation. According to a criticism put forward in Ref. [10], inflating regions cannot be said to dominate the volume of the universe because the volume is not a gauge-invariant characteristic. One might then ask whether the entire picture of eternal inflation is not a gauge artifact or a result of inconsistently applied statistics. For instance, it was found in Ref. 11] that certain volume-weighted observables behave quite similarly in models with and without eternal inflation, thus casting doubt on the usually claimed ability of eternal inflation to forget initial conditions.

The purpose of this paper is to answer these criticisms and to describe the defining features of eternal inflation more accurately. As I will discuss below, the mentioned criticisms correctly point out the flaws in certain often made statements about eternal inflation. Nevertheless, the standard picture of eternal inflation remains unchanged. The presence of eternal inflation is manifested 
by the permanent increase of the number of causally disconnected non-thermalized regions. On the other hand, answers to certain other questions, especially those involving the proper 3-volume, turn out to be heavily influenced by the choice of equal-time surfaces. I shall attempt to clarify the influence of the gauge by analyzing various specific statements that might be made about observables in eternally inflating spacetimes.

The following questions will be answered:

- Whether the volume of inflating regions is larger than the volume of thermalized regions. (Depends on the choice of the time gauge.)

- More generally, whether a volume-weighted value of an observable has a qualitatively different behavior when eternal inflation occurs. (Depends on the choice of the time gauge.)

- Whether it is possible to describe the manifestations of eternal inflation in a gauge-invariant manner. In particular, whether the volume of the inflating domain exhibits an unbounded growth with time regardless of the time gauge. (Yes, no.)

- Whether the FP formalism admits a particular time gauge such that the resulting probability distribution is unbiased. (No.)

\section{ETERNAL INFLATION IN A BOX}

The global geometry of eternally inflating spacetimes possesses certain counter-intuitive properties. To render the discussion more visual, let us turn to a drastically simplified toy model of eternal inflation that nevertheless exhibits all the qualitative features of interest.

In the toy model which we call "inflation in a box," time elapses in discrete steps $s=0,1,2, \ldots$ and the space is reduced to two dimensions and further to the square domain $0<x, y<1$, where $x, y$ are the comoving coordinates measured in Hubble units $H^{-1}$. The entire initial Hubble-size domain is assumed to be inflating at time $s=0$. To imitate inflation during one timestep, we subdivide the initial inflating square into $N \times N$ equal sub-squares of size $N^{-1} \times N^{-1}$; at the next step, each sub-square will again have the Hubble proper size. Then we randomly mark some of the smaller squares as "thermalized" assuming that each Hubble-size inflating square continues inflation with a probability $q$ (where $0<q<1$ ) and thermalizes with probability $1-q$, independently of all other squares. The selection of thermalized squares concludes the simulation for one timestep. At the next timestep, the same procedure of subdivision and random thermalization is applied to each Hubble-sized inflating square, while the "thermalized" squares do not evolve any further (see Fig. 1). This random process was called a "random Sierpiński carpet" or a "curdling" in the book [12].

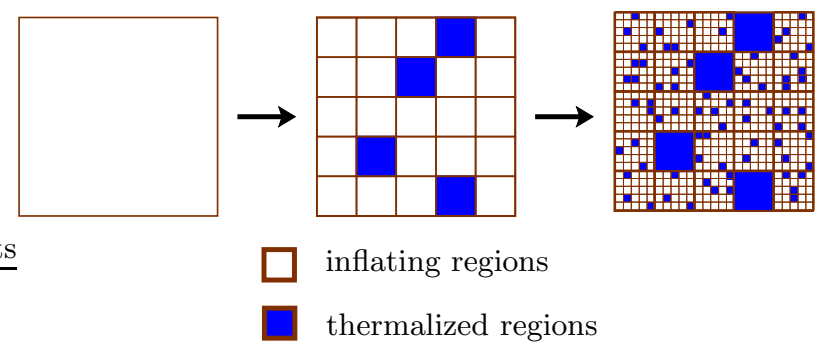

Figure 1: First steps in the construction of a random Sierpiński carpet with $N=5$ and $q=5 / 6$.

At a step $s$ we thus have a number of inflating squares with comoving size $N^{-s} \times N^{-s}$ and also a number of thermalized squares whose comoving sizes are determined by the times of their thermalization. It is straightforward to show that a given comoving point $(x, y)$ remains in the inflating regime with probability $q^{s}$ after $s$ steps, and that the mean number of inflating squares at a step $s$ is $\left(N^{2} q\right)^{s}$ while the mean comoving 2-volume of the inflating domain is $q^{s}$. Since each inflating square has Hubble proper size, the number of inflating squares $\left(N^{2} q\right)^{s}$ is naturally interpreted as the proper (as opposed to comoving) volume of the inflating domain at time $s$. It follows that the mean proper volume of inflating domain grows without bound if $N^{2} q>1$ and decreases to zero if $N^{2} q<1$. Thus the condition $N^{2} q>1$ allows futureeternal inflation to occur with a nonzero probability. (A rigorous derivation of these results is given in the theory of branching processes, see e.g. the book [13], chapter 1.)

This toy model serves as a crude analogy for the process of random nucleation of bubbles in a de Sitter spacetime (see e.g. [14]). The "thermalized squares" represent the nucleated bubbles of true vacuum which expand and quickly reach the comoving Hubble size (computed at nucleation time). The subdivision of the inflating squares mimics an exponential expansion, while the parameter $N$ plays the role of the expansion factor during one timestep. Since the thermalized squares are not further subdivided, the expansion of spacetime within the true vacuum bubbles is not modeled. As a remedy we consider a variation of the box model where the thermalized squares remain thermalized but are subdivided into $M \times M$ parts at each timestep, where $M<N$ is chosen to emulate the slower expansion of true vacuum regions. This variation of the box model characterized by the parameters $q, N, M$ will be sufficiently general for the present analysis.

Let us now make a connection of the box model with models of eternal inflation driven by a scalar field $\phi$ with an effective potential $V(\phi)$. The FP formalism requires to choose a time slicing parametrized by a time coordinate $\tau$. A possible choice is $\tau=t$ where $t$ is the proper time measured along comoving geodesics $\mathbf{x}=$ const. Then one considers two probability distributions defined at a fixed time $\tau$ : first, the distribution $P(\phi, \tau)$ of values along a given (randomly chosen) comoving trajectory, and sec- 
ond, the total proper volume $P_{V}(\phi, \tau)$ of all regions with the value $\phi$ at time $\tau$. (Some issues regarding the interpretation of the distributions $P$ and $P_{V}$ are clarified in Appendix A) If the time parameter $\tau$ is related to the proper time $t$ by a function of $\phi$ only,

$$
d \tau=T(\phi) d t, \quad T(\phi)>0,
$$

then one can compute the probability distributions $P$ and $P_{V}$ by solving the FP equations, ${ }^{2}$

$$
\begin{aligned}
\partial_{\tau} P & =\partial_{\phi}^{2}(D(\phi) P)-\partial_{\phi}(v(\phi) P), \\
\partial_{\tau} P_{V} & =\partial_{\phi}^{2}\left(D(\phi) P_{V}\right)-\partial_{\phi}\left(v(\phi) P_{V}\right)+3 h(\phi) P_{V},
\end{aligned}
$$

where $D(\phi), v(\phi)$, and $h(\phi)$ are appropriate kinetic coefficients [16, 17] that depend on the potential $V(\phi)$ and on the function $T(\phi)$. The precise form of these coefficients will not be used in this paper.

We can describe "inflation in a box" by analogous equations if we assume that the variable $\phi$ takes on a discrete set of values, e.g. $\phi=0$ standing for inflation and $\phi=1$ for thermalization. The distributions $P(\phi, \tau)$ and $P_{V}(\phi, \tau)$ are reduced to $P_{j}(s) \equiv P(j, s)$ and $P_{V j}(s) \equiv P_{V}(j, s)$, where $j=0,1$ and $s$ is the timestep. The equations describing these distributions are

$$
\begin{aligned}
P_{0}(s+1) & =q P_{0}(s), \\
P_{1}(s+1) & =P_{1}(s)+(1-q) P_{0}(s), \\
P_{V 0}(s+1) & =N^{2} q P_{V 0}(s), \\
P_{V 1}(s+1) & =M^{2} P_{V 1}(s)+N^{2}(1-q) P_{V 0}(s),
\end{aligned}
$$

where $q, N, M$ are the parameters of the box model. With the initial conditions $P_{0}(0)=P_{V 0}(0)=1, P_{1}(0)=$ $P_{V 1}(0)=0$, the solutions are

$$
\begin{gathered}
P_{0}(s)=q^{s}, P_{V 0}(s)=\left(N^{2} q\right)^{s}, \quad P_{1}(s)=1-q^{s}, \\
P_{V 1}(s)=\frac{1-q}{q}\left(N^{2} q\right)^{s} \frac{1-\lambda^{s}}{1-\lambda}, \quad \lambda \equiv \frac{M^{2}}{N^{2} q} .
\end{gathered}
$$

It is clear that the proper volume of the inflating domain $P_{V 0}(s)$ grows without bound if $N^{2} q>1$. This is the usual sign of eternal inflation.

It will be more convenient to analyze the box model in continuous time $t$ rather than in the discrete time $s$. Denoting by $\alpha$ the thermalization rate and by $H_{0}, H_{1}$ the expansion rates of the inflating and the thermalized domains respectively, we can write the following equations describing the distributions of the comoving and the proper volume,

$$
\begin{aligned}
\frac{d}{d t} P_{0} & =-\alpha P_{0}, \quad \frac{d}{d t} P_{1}=\alpha P_{0}, \\
\frac{d}{d t} P_{V 0} & =2 H_{0} P_{V 0}-\alpha P_{V 0}, \\
\frac{d}{d t} P_{V 1} & =2 H_{1} P_{V 1}+\alpha P_{V 0} .
\end{aligned}
$$

\footnotetext{
${ }^{2}$ Here and below we use the Ito factor ordering in the diffusion terms [15].
}

With the same initial conditions as above, the solution is

$$
\begin{gathered}
P_{0}(t)=e^{-\alpha t}, P_{V 0}(t)=e^{\left(2 H_{0}-\alpha\right) t}, \\
P_{1}(t)=1-e^{-\alpha t}, P_{V 1}(t)=\frac{e^{\left(2 H_{0}-\alpha\right) t}-e^{2 H_{1} t}}{2\left(H_{0}-H_{1}\right)-\alpha} \alpha .
\end{gathered}
$$

The correspondence with the discrete-time model is obtained by setting $q=e^{-\alpha}$ and $H_{0}=\ln N, H_{1}=\ln M$. Eternal inflation is possible if $\alpha<2 H_{0}$.

An example of a different choice of the time variable is the "e-folding time" $\tau=\ln a$, where $a$ is the scale factor. To derive the analogs of Eqs. (10)-(12) for the volume distributions at constant $\tau$, we note that an infinitesimal interval $d \tau$ corresponds to the proper time interval $d t=H_{0}^{-1} d \tau$ within the inflating domains, and thus a fraction $\alpha d t=\alpha H_{0}^{-1} d \tau$ of the total inflating volume will thermalize during the interval $d \tau$. This applies to the comoving as well as to the proper volume since the inflating domain is homogeneous. Therefore the increase $d P_{1}$ in the comoving volume of the thermalized domain during the $e$-folding time interval $d \tau$ will be $\alpha H_{0}^{-1} P_{0} d \tau$. In this way we arrive to the equations

$$
\begin{aligned}
\frac{d}{d \tau} P_{0} & =-\frac{\alpha}{H_{0}} P_{0}, \quad \frac{d}{d \tau} P_{1}=\frac{\alpha}{H_{0}} P_{0}, \\
\frac{d}{d \tau} P_{V 0} & =2 P_{V 0}-\frac{\alpha}{H_{0}} P_{V 0} \\
\frac{d}{d \tau} P_{V 1} & =2 P_{V 1}+\frac{\alpha}{H_{0}} P_{V 0} .
\end{aligned}
$$

Note that these equations cannot be obtained from Eqs. (101)-(12) by a simple change of the time variable. The solutions of Eqs. (15)-(17) are

$$
\begin{gathered}
P_{0}(\tau)=e^{-\alpha \tau / H_{0}}, P_{V 0}(\tau)=e^{\left(2-\alpha / H_{0}\right) \tau}, \\
P_{1}(\tau)=1-e^{-\alpha \tau / H_{0}}, P_{V 1}(\tau)=e^{2 \tau}-e^{\left(2-\alpha / H_{0}\right) \tau} .
\end{gathered}
$$

The volume of the inflating domain grows proportionally to $a^{2-\alpha / H_{0}}$ in both the time gauges because the inflationary expansion rate $H_{0}$ is everywhere the same. A two-dimensional domain whose proper 2 -volume grows slower than $a^{2}$, namely as $a^{\gamma}$ with $\gamma<2$, can be visualized as an expanding lacunary fractal with dimension $\gamma$, in the following sense. A lacunary fractal set $S$ having a fractal dimension $\gamma<2$ contains infinitely many "holes" of diminishing size, and the 2 -volume of $S$ vanishes. To obtain a domain with a nonvanishing volume, one can coarse-grain the set $S$ on a fixed scale which is the Hubble scale in the context of inflation. We may denote the result of the coarse-graining by $\tilde{S}$; the set $\tilde{S}$ is interpreted as the inflating domain and the holes in $S$ are regions that will eventually thermalize. At a given time, holes in $S$ having sizes below the Hubble scale are invisible in the coarse-grained set $\tilde{S}$. As the set $S$ expands (while the coarse-graining scale is kept fixed), it appears that new holes are constantly created within the domain $\tilde{S}$, making its volume grow slower than $a^{2}$. The resulting fractal structure of the inflating domain was first explored in 
Ref. [18] where it was shown that a domain expanding as $\propto a^{\gamma}$ is characterized by the fractal dimension $\gamma$. Thus the fractal dimension of the inflating domain in the box model is $\gamma=2-\alpha H_{0}^{-1}$. A more rigorous definition of the fractal dimension of the inflating domain, together with a proof of its gauge independence, was given in Ref. [17].

\section{VOLUME OF INFLATING VS. THERMALIZED DOMAINS}

Let us now consider the question of whether the inflating domain dominates the volume of the universe at late times. In the box model with continuous time, the ratio of the total thermalized volume to the total inflating volume at a proper time $t$ is found from Eqs. (13)-(14),

$$
\left.\frac{V_{\text {therm }}}{V_{\text {infl }}}\right|_{s}=\frac{P_{V 1}(t)}{P_{V 0}(t)}=\alpha \frac{1-e^{-\mu t}}{\mu}, \quad \mu \equiv 2\left(H_{0}-H_{1}\right)-\alpha .
$$

At late times $t \rightarrow \infty$, this ratio tends to infinity if $\mu \leq 0$ and to a finite nonzero limit $\alpha / \mu$ if $\mu>0$. The value of the latter limit may be any real number depending on the values of the parameters $H_{0}, H_{1}, \alpha$. With the typical assumptions $\alpha \ll 2 H_{0}$ and $H_{1}<H_{0}$, we find a finite and small value of the limit, $\alpha / \mu \ll 1$, indicating that most of the volume is in the inflating domain.

However, if we compute the volume ratio at a fixed scale factor rather than at a fixed step, we obtain a different result,

$$
\left.\frac{V_{\text {therm }}}{V_{\text {infl }}}\right|_{\tau}=\frac{P_{V 1}(\tau)}{P_{V 0}(\tau)}=e^{\alpha \tau / H_{0}}-1
$$

This ratio tends to infinity as $\tau \rightarrow \infty$, indicating that most of the volume is contained in the thermalized domain, in a direct opposition to the typical result obtained in the proper time gauge. Therefore the statement that the volume is dominated by the inflating domain during eternal inflation does not hold in all gauges. Also, the ratio of the inflating to the thermalized volume does not necessarily characterize the presence or the absence of eternal inflation.

\section{VOLUME-WEIGHTED OBSERVABLES}

We now consider volume-weighted observables in an eternally inflating spacetime. A volume-weighted observable is defined using the volume $V(A)$ occupied by regions that have a certain value $A$ of the observable at a time $t$ :

$$
\langle A\rangle_{V}=\frac{\sum_{A} A V(A)}{\sum_{A} V(A)} .
$$

Volume-weighting is always performed along a particular equal-time surface.

In the box model, the possible candidates for volume weighting are the field $\phi$ and the scale factor $a$. Since the field $\phi$ serves only to indicate the inflating or the thermalized state of a region, a volume-weighted average of $\phi$ or, more generally, of a function $A(\phi)$ at a time $t$ is completely determined by the distributions $P_{V 0}(t)$ and $P_{V 1}(t)$, i.e.

$$
\langle A(\phi)\rangle_{V}=\frac{A(0) P_{V 0}+A(1) P_{V 1}}{P_{V 0}+P_{V 1}}=\frac{A(0)+A(1) \frac{P_{V 1}}{P_{V 0}}}{1+\frac{P_{V 1}}{P_{V 0}}} .
$$

As we have seen, the ratio $P_{V 1} / P_{V 0}$ is highly gaugedependent and so are the volume-weighted averages of $\phi$ or of any functions of $\phi$.

Turning now to the scale factor $a$, we first note that in the scale factor gauge the entire equal-time hypersurface has the same value of $a$ and it is useless to perform a volume-weighted averaging. Therefore we consider the proper time gauge, where the volume-weighted average of the scale factor differs from its statistical mean. To describe the distribution of the scale factor observed at a proper time $t$ at a given comoving point in the box model, it suffices to compute the probability density $p_{t h}(s)$ for the given point to thermalize within a time interval $[s, s+$ $d s]$,

$$
p_{t h}(s) d s=e^{-\alpha s} \alpha d s .
$$

It is clear that $p_{t h}(s)$ is the also the probability density for the scale factor to assume the value $a=e^{H_{0} s+H_{1}(t-s)}$ at time $t \geq s$ at a randomly chosen comoving point. It is more convenient to perform computations with the number of $e$-foldings, $\ln a$, for which we have the statistical mean

$$
\langle\ln a\rangle=\left\langle s H_{0}+(t-s) H_{1}\right\rangle=H_{1} t+\left(H_{0}-H_{1}\right)\langle s\rangle
$$

and the volume-weighted mean

$$
\langle\ln a\rangle_{V}=H_{1} t+\left(H_{0}-H_{1}\right)\langle s\rangle_{V}
$$

so it remains to compute the means $\langle s\rangle$ and $\langle s\rangle_{V}$ using the distribution $p_{t h}(s)$. Calculations yield

$$
\langle s\rangle=\int_{0}^{t} p_{t h}(s) s d s=\frac{1-e^{-\alpha t}}{\alpha}-t e^{-\alpha t}
$$

and

$$
\langle s\rangle_{V}=\frac{\int_{0}^{t} p_{t h}(s) e^{2 H_{0} s+2 H_{1}(t-s)} s d s}{\int_{0}^{t} p_{t h}(s) e^{2 H_{0} s+2 H_{1}(t-s)} d s}=\frac{\mu t e^{\mu t}-e^{\mu t}+1}{\left(e^{\mu t}-1\right) \mu},
$$

where $\mu$ is defined in Eq. (20). Depending on the value of the parameter $\mu$, the volume-weighted average for late times $t$ may be dominated by the contribution of either inflating or thermalized regions. For instance, if $\mu \leq 0$ (domination by thermalized regions) then

$$
\lim _{t \rightarrow \infty}\langle s\rangle_{V}=-\frac{1}{\mu}
$$


and thus for large $t$ we have

$$
\langle\ln a\rangle_{V}=H_{1} t-\frac{1}{\mu}\left(H_{0}-H_{1}\right) \approx H_{1} t,
$$

which is almost the same as the statistical mean,

$$
\langle\ln a\rangle=H_{1} t+\frac{1}{\alpha}\left(H_{0}-H_{1}\right)
$$

On the other hand, if $\mu>0$ (domination by inflating regions) then $\langle s\rangle_{V} \approx t$, i.e. almost all volume is filled by very recently thermalized regions, and so

$$
\langle\ln a\rangle_{V} \approx H_{1} t+\left(H_{0}-H_{1}\right) t=H_{0} t .
$$

Note that the possibility of eternal inflation is compatible with both cases $\mu>0$ and $\mu \leq 0$. We conclude that volume-weighted observables such as $\langle\ln a\rangle_{V}$ generally furnish neither any information specific to eternal inflation nor any gauge-invariant information.

\section{GROWTH OF VOLUME OF INFLATING DOMAINS}

The hallmark of eternal inflation is the unbounded increase in the total number of independent inflating regions. It is also often stated that the total proper 3volume of the inflating domain grows without bound at late times in an eternally inflating spacetime; this is certainly true for the 3-volume computed along hypersurfaces of equal proper time or of equal scale factor. One of the criticisms expressed in Refs. [10, 11] was that the 3 -volume is a gauge-dependent characteristic and thus cannot be used as an indication of the presence or the absence of eternal inflation. In an earlier attempt to resolve this issue, it was proven in Ref. [17] (Sec. III A) that an eternally inflating spacetime manifests an unbounded volume growth in all time gauges of the form (11). However, one may imagine time gauges not of the form (11). Equal-time probability distributions in such gauges cannot be described by the FP equations (2)-(3) where the kinetic coefficients depend only on the field $\phi$.

In fact, the statement about the growing volume of the inflating domain is gauge-dependent. If one allows completely arbitrary time slicings, the total volume of the inflating domain during eternal inflation might either grow or decrease depending on the time slicing. I illustrate this dramatic dependence on the choice of gauge in Appendix B where I present an explicitly constructed foliation of a de Sitter spacetime by spacelike hypersurfaces whose proper 3 -volume monotonically decreases to zero at late times. These hypersurfaces are artificially chosen to be almost null almost everywhere, which makes their 3-volume arbitrarily small despite the expansion of the background spacetime. Since an inhomogeneous eternally inflating universe expands "slower" than a pure de Sitter universe, the same family of hypersurfaces will also have a decreasing 3 -volume in inhomogeneous inflating universes. It follows that the 3-volume of an arbitrary family of equal-time hypersurfaces cannot be used as a criterion for the presence of eternal inflation.

However, a weaker statement is sufficient: namely, eternal inflation is present if (and only if) there exists a choice of time slicing with an unbounded growth of the 3volume of inflating domains. In the remainder of this section we shall demonstrate the equivalence of this criterion and the usual definition of eternal inflation (the existence of inflating domains at arbitrary late times). Note that the existence of something "at an arbitrarily late time" is a gauge-independent notion due to the monotonicity of time parameters in all gauges.

If the 3 -volume of the inflating domain grows without bound in some time gauge, then obviously there exist inflating domains at arbitrarily late times. It remains to prove that if inflating domains exist arbitrarily late, then the inflating volume grows in some gauge. This growth can be most easily demonstrated in the $e$-folding gauge, $\tau=\ln a$, using the construction of "eternal comoving points" 17]. These points follow comoving geodesic worldlines that forever remain within the inflating domain and never enter any thermalized regions. It was shown in Ref. 17] using general topological arguments that the presence of inflating domains at arbitrarily late times entails the existence of infinitely many such "eternal points." Since each eternal point is surrounded by at least a Hubble-sized inflating region at any time, the 3 -volume of the inflating domain at $e$-folding time $\tau$ is greater than $H^{-3} N$, where $N$ is the number of eternal points that are pairwise separated by a proper distance of at least $H^{-1}$ at time $\tau$. To show that the volume is greater than a preassigned bound $M$, we thus need to find at least $N_{0}=M H^{3}$ widely separated eternal points at a sufficiently late time. Since the eternal points are infinitely numerous, we can always choose a subset $S$ containing $N_{0}$ such points and then determine the minimum comoving distance $\delta x$ between any two points from the subset $S$. Within a hypersurface of constant scale factor $a=e^{\tau}$, the proper Hubble distance $H^{-1}$ corresponds to the comoving distance $H^{-1} e^{-\tau}$. For sufficiently late $e$-folding times $\tau>\tau_{0} \equiv-\ln (H \delta x)$ the scale factor $a=e^{\tau}$ is large enough so that the proper distance between any two points from $S$ is larger than $H^{-1}$. Thus for $\tau>\tau_{0}$ there exist at least $N_{0}$ independent inflating regions, each having a volume at least $H^{-3}$. So we have shown that the volume of the inflating domain for $\tau>\tau_{0}$ is greater than $N_{0} H^{-3}=M$. Since $M$ is an arbitrary bound, we conclude that the 3 -volume computed in the $e$-folding gauge grows without bound. It follows that an unbounded growth of the 3 -volume is found also in all gauges of the form (1), including the proper time gauge. 


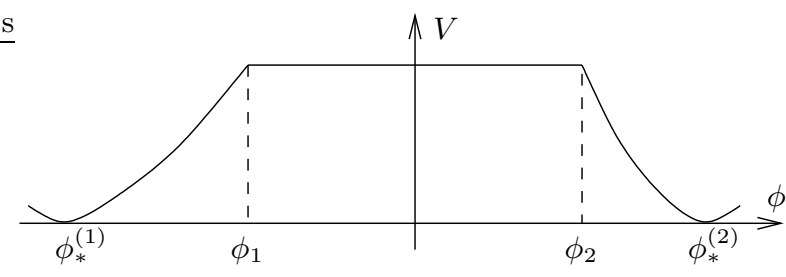

Figure 2: Illustrative inflationary potential with a flat selfreproduction regime $\phi_{1}<\phi<\phi_{2}$ and deterministic regimes $\phi_{*}^{(1)}<\phi<\phi_{1}$ and $\phi_{2}<\phi<\phi_{*}^{(2)}$.

\section{IS THERE A "CORRECT" TIME PARAMETER?}

As we have seen in Sec. III the division of 3-volume between the inflating and the thermalized domains is strongly gauge-dependent. For instance, the proper-time distribution is biased towards regions with faster expansion rates, while the scale factor gauge favors regions with larger comoving volumes. The probability distribution $P(\phi, \tau)$ necessarily depends on the choice of the time parameter $\tau$. It has been sometimes proposed that a certain choice of the parameter $\tau$ will yield physically justified, unbiased probability distributions. In this section I shall demonstrate that no choice of the time parameter guarantees unbiased results, at least in a certain class of inflationary models.

In the relevant class of models, the self-reproduction regime is symmetric with respect to the observable parameters; a specific model of this kind was considered in Ref. [7]. For the present purposes it is sufficient to analyze a toy model of scalar-field inflation with an effective potential shown in Fig. 22 Our analysis shall partially follow Ref. [6] where a similar potential was introduced. The potential is flat in the range $\phi_{1}<\phi<\phi_{2}$ where the evolution is fluctuation-dominated, while the evolution of regions with $\phi>\phi_{2}$ or $\phi<\phi_{1}$ is completely deterministic (fluctuation-free). It is assumed that the fluctuation-dominated range $\phi_{1}<\phi<\phi_{2}$ is sufficiently wide to cause an eternal self-reproduction of inflating regions. There are two thermalization points, $\phi=\phi_{*}^{(1)}$ and $\phi=\phi_{*}^{(2)}$, which may be associated to different types of true vacuum and thus to different observed values of cosmological parameters. The question is to compare the volumes $\mathcal{V}_{1,2}$ of regions thermalized into these two vacua. Since there is an infinite volume thermalized into either vacuum, one hopes to obtain a sensible answer for the volume ratio $\mathcal{V}_{1} / \mathcal{V}_{2}$.

In the present case, due to the symmetry of the potential it is natural to assume that Hubble-sized regions exiting the eternal self-reproduction regime at $\phi=\phi_{1}$ and at $\phi=\phi_{2}$ are equally abundant. Since the evolution within the ranges $\phi_{*}^{(1)}<\phi<\phi_{1}$ and $\phi_{2}<\phi<\phi_{*}^{(2)}$ is deterministic, the regions exiting the self-reproduction regime at $\phi=\phi_{1}$ or $\phi=\phi_{2}$ will experience a fixed num- ber of $e$-foldings which we may denote $\ln Z_{1}$ and $\ln Z_{2}$ respectively. Therefore the volume of regions thermalized at $\phi=\phi_{*}^{(j)}$, where $j=1,2$, will be increased by the factors $Z_{j}^{3}$ and thus the volume ratio is

$$
\frac{\mathcal{V}_{1}}{\mathcal{V}_{2}}=\frac{Z_{1}^{3}}{Z_{2}^{3}}
$$

We shall now compare this result with that of the gauge-dependent FP approach. The time-dependent distribution of the 3 -volume $P_{V}(\phi, \tau)$ is a solution of the FP equation (3) in a time gauge $\tau$. The late-time behavior of the volume distribution is

$$
P_{V}(\phi, \tau)=P_{V}^{(0)}(\phi) e^{\gamma \tau},
$$

where $\gamma>0$ is the largest eigenvalue of the corresponding stationary FP equation. (It was shown in Ref. 17 that $\gamma>0$ in all gauges $\tau$ admitted by the FP formalism.) Since the volume of regions with all values of $\phi$ grows without bound, one needs to introduce a cutoff to compare the total volumes $\mathcal{V}_{1,2}$ thermalized at $\phi=\phi_{*}^{(1)}$ and $\phi=\phi_{*}^{(2)}$. The equal-time cutoff, i.e. the procedure where one counts only the volume of the regions thermalized before a fixed time $\tau=\tau_{\max }$, is implemented straightforwardly in the FP formalism and in the present case yields $\underline{6}$ ]

$$
\frac{\mathcal{V}_{1}}{\mathcal{V}_{2}}=\frac{Z_{1}^{3}}{Z_{2}^{3}} \exp \left[-\gamma\left(\Delta \tau_{1}-\Delta \tau_{2}\right)\right],
$$

where $\Delta \tau_{j}, j=1,2$, are the time intervals elapsed in the time gauge $\tau$ during the deterministic evolution of the field from $\phi_{j}$ to $\phi_{*}^{(j)}$.

One can derive Eq. (35) from elementary considerations as follows. A Hubble region exiting the selfreproduction regime at $\phi=\phi_{1}$ will thermalize after a fixed duration of time $\Delta \tau_{1}$ and will accumulate the growth factor $Z_{1}$ during this time. Similarly, regions exiting at $\phi=\phi_{2}$ will reach the thermalization point $\phi=\phi_{*}^{(2)}$ after a time interval $\Delta \tau_{2}$ and accumulate the growth factor $Z_{2}$. The "arrival times" $\Delta \tau_{j}$ and the growth factors $Z_{j}, j=1,2$, can be computed using the particular shape of the potential $V(\phi)$ but we shall not need their explicit forms.

It follows that the regions thermalized at $\phi=\phi_{*}^{(j)}$ at time $\tau$ have previously exited the self-reproduction regime at time $\tau-\Delta \tau_{j}$. According to Eq. (34), the volume of regions exiting at $\phi=\phi_{j}$ at time $\tau-\Delta \tau_{j}$ is

$$
P_{V}^{(0)}\left(\phi_{j}\right) \exp \left[\gamma\left(\tau-\Delta \tau_{j}\right)\right],
$$

where $P_{V}^{(0)}\left(\phi_{1}\right)=P_{V}^{(0)}\left(\phi_{2}\right) \equiv P_{V}^{(0)}$ due to the symmetry of the potential in the self-reproduction regime. The total volume of regions thermalized at $\phi=\phi_{*}^{(j)}$ up to a time $\tau_{\max }$ is then

$$
\begin{aligned}
\mathcal{V}_{j}\left(\tau_{\max }\right) & =P_{V}^{(0)} Z_{j}^{3} \int_{0}^{\tau_{\max }} \exp \left[\gamma\left(\tau-\Delta \tau_{j}\right)\right] \\
& \propto Z_{j}^{3} \exp \left[\gamma\left(\tau_{\max }-\Delta \tau_{j}\right)\right]
\end{aligned}
$$


where we have omitted $j$-independent factors. Thus we obtain the volume ratio as the limit

$$
\frac{\mathcal{V}_{1}}{\mathcal{V}_{2}}=\lim _{\tau_{\max } \rightarrow \infty} \frac{\mathcal{V}_{1}\left(\tau_{\max }\right)}{\mathcal{V}_{2}\left(\tau_{\max }\right)}=\frac{Z_{1}^{3}}{Z_{2}^{3}} \exp \left[-\gamma\left(\Delta \tau_{1}-\Delta \tau_{2}\right)\right]
$$

This coincides with Eq. (35).

As already noted in Ref. 6], the reason for the gauge dependence of the volume ratio (35) is the presence of the times $\Delta \tau_{j}$ which generally leads to an appreciable bias. The arrival times $\Delta \tau_{j}$ are generally different, $\Delta \tau_{1} \neq \Delta \tau_{2}$, unless the inflaton potential $V(\phi)$ is completely symmetric. ${ }^{3}$ Since $\gamma>0$ in all gauges, it is impossible to choose a gauge $\tau$ in which the results (33) and (35) would coincide for all potentials. We conclude that there exists no "correct" gauge $\tau$ that would always guarantee unbiased results for the equal-time cutoff procedure.

\section{Acknowledgments}

The author is grateful to Matthew Parry for stimulating conversations and to Alex Vilenkin for discussions and comments on the manuscript. Thanks are also due to the referee who made some useful suggestions.

\section{Appendix A: INTERPRETATIONS OF THE FOKKER-PLANCK FORMALISM}

In this appendix I shall clarify the possible interpretations of the distributions $P(\phi, \tau)$ and $P_{V}(\phi, \tau)$ in terms of an ensemble of imaginary comoving observers placed throughout the inflationary spacetime.

The comoving volume distribution $P(\phi, \tau)$ can be found as a solution of the FP equation (2) as well as via a Langevin equation. The Langevin and the FokkerPlanck equations are equivalent descriptions of the same stochastic process, and we shall focus on the FP description.

The distribution $P(\phi, \tau)$ allows two interpretations. On the one hand, $P(\phi, \tau)$ is the probability for the field to have a value $\phi$ at time $\tau$ along a single, randomly chosen comoving trajectory; on the other hand, $P(\phi, \tau)$ is the fraction of comoving volume where the field has the value $\phi$, within an equal- $\tau$ hypersurface. (The "comoving 3 -volume" of a subdomain $S$ of an equal- $\tau$ hypersurface is defined as the 3 -volume of the subdomain $S_{0}$ of the hypersurface $\tau=0$, where $S_{0}$ consists of points whose comoving worldlines pass through $S$ at time $\tau$.)

\footnotetext{
3 The arrival times would be equal if it were possible to use the hypersurfaces of equal $\phi$ as the time slicing. However, a hypersurface $\phi=$ const is in general not spacelike because the evolution of $\phi$ is not monotonic in the self-reproduction regime.
}

The equivalence of these interpretations can be seen from the following argument. Consider a large but finite domain of volume $V_{0}$ within the hypersurface $\tau=0$ and a very dense grid of comoving trajectories starting out from this domain. The total number $N$ of these trajectories may be chosen as finite but very large, and the initial volume $V_{0}$ can be subdivided into $N$ tiny regions of comoving volume $V_{0} / N$ situated around each of the $N$ comoving lines. If the initial volume $V_{0}$ is sufficiently large so that all the statistically possible histories are sufficiently well sampled, then the number of lines that reach a value $\phi$ at a time $\tau$ is very close to $N P(\phi, \tau)$. Thus the comoving volume of the subdomain of the equal- $\tau$ hypersurface having the value $\phi$ is $V_{0} P(\phi, \tau)$. Therefore in the statistical limit $N \rightarrow \infty$ and $V_{0} \rightarrow \infty$, the fraction of the comoving volume containing the field value $\phi$ at the time $\tau$ is equal to $P(\phi, \tau)$.

Conversely, if $P(\phi, \tau)$ is the statistical distribution of the comoving volume along an equal- $\tau$ hypersurface, then the comoving volume of the subdomain with the value $\phi$ will be $V_{0} P(\phi, \tau)$. We denote this subdomain by $S(\phi, \tau)$ and consider a comoving trajectory randomly chosen from the total comoving volume $V_{0}$. The probability of this trajectory passing through the subdomain $S(\phi, \tau)$ is equal to $V_{0} P(\phi, \tau) / V_{0}=P(\phi, \tau)$. Therefore $P(\phi, \tau)$ is the probability of the field having the value $\phi$ at time $\tau$ along a randomly chosen trajectory.

We note that there do exist correlations between the values of $\phi$ at nearby comoving trajectories. However, these correlations are not reflected by the distribution $P(\phi, \tau)$ since it specifies only the total volume, but not the location, of regions with the field value $\phi$. If the initial comoving volume $V_{0}$ is sufficiently large (many Hubble volumes), the distribution of the field $\phi$ at time $\tau$ along a randomly chosen comoving trajectory will be $P(\phi, \tau)$ despite the correlations between nearby trajectories.

Turning now to the distribution of the proper volume $P_{V}(\phi, \tau)$, we note that there is now only one interpretation, namely $P_{V}(\phi, \tau)$ is the total proper 3 -volume of the subdomain with the field value $\phi$ within an equal- $\tau$ hypersurface. (As before, we consider only the comoving future of a large but finite initial volume $V_{0}$ at time $\tau=0$.) It appears to be impossible to interpret $P_{V}(\phi, \tau)$ directly in terms of observations made on a single randomly chosen comoving trajectory. The distribution $P_{V}(\phi, \tau)$ describes a branching diffusion process with a possibly unlimited growth, and its interpretation necessarily has to involve a potentially very large ensemble of trajectories.

A connection can be formulated between the distribution $P_{V}(\phi, \tau)$ and the joint comoving distribution of the field and the scale factor $P(\phi, a, \tau)$. The comoving distribution $P(\phi, a, \tau)$ is interpreted as the fraction of the comoving volume where the field has the value $\phi$ and the scale factor has the value $a$, within an equal- $\tau$ hypersurface. Alternatively, $P(\phi, a, \tau)$ is the probability of the field having the value $\phi$ and the scale factor having the value $a$ at time $\tau$ along a single, randomly chosen comoving trajectory. The distribution $P(\phi, a, \tau)$ is the solution 
of the FP equation

$$
\partial_{\tau} P=\hat{L}_{\phi} P-\partial_{a}(h(\phi) a P),
$$

where

$$
\hat{L}_{\phi} P \equiv \partial_{\phi}^{2}(D(\phi) P)-\partial_{\phi}(v(\phi) P)
$$

is the differential operator entering Eq. (2) and $h(\phi)$ is the "expansion rate" function defined by

$$
\frac{d a}{d \tau}=h(\phi) a
$$

For example, in the proper-time gauge $h(\phi)=H(\phi)$ while in the $e$-folding gauge $h \equiv 1$.

To obtain the distribution $P_{V}(\phi, \tau)$, one can use the procedure of "volume-weighting,"

$$
P_{V}(\phi, \tau) \equiv \int_{0}^{\infty} d a a^{3} P(\phi, a, \tau),
$$

which takes into account the expansion factor $a^{3}$ along the trajectory. It is easy to verify that the resulting (unnormalized) distribution $P_{V}(\phi, \tau)$ satisfies the FP equation (3). This establishes a link between the volume interpretation of the distribution $P_{V}(\phi, \tau)$ and properties of a single comoving trajectory embodied by the joint probability $P(\phi, a, \tau)$.

\section{Appendix B: COUNTEREXAMPLES TO THE GROWTH OF VOLUME}

In this section I shall present two examples of foliations described by spacelike hypersurfaces

$$
t=t(\tau, \mathbf{x}), \quad-\infty<\tau<+\infty,
$$

such that the total proper 3-volume of each equal- $\tau$ hypersurface is finite and monotonically decreases with $\tau$, asymptotically approaching zero at late times. The two examples will be foliations of the Minkowski spacetime and of the future part of a de Sitter spacetime, the latter obviously interpreted as a homogeneous, eternally inflating universe. These examples unequivocally demonstrate that an unbounded growth of the 3-volume of equal-time hypersurfaces during eternal inflation is not observed in all gauges.

I shall first describe the idea behind the construction of the foliations and later give technical details. For simplicity, let us begin by considering a flat $1+1$-dimensional spacetime with standard coordinates $(t, x)$ and a sawshaped hypersurface

$$
t=S_{0}(x) \equiv \frac{L}{\pi} \cos ^{-1}\left(\cos \frac{\pi x}{L}\right),
$$

where the inverse cosine function is by definition such that $0 \leq S_{0}(x) \leq L$. The hypersurface $t=S_{0}(x)$ is piecewise null and has kinks at points $x=n L, n \in \mathbb{Z}$

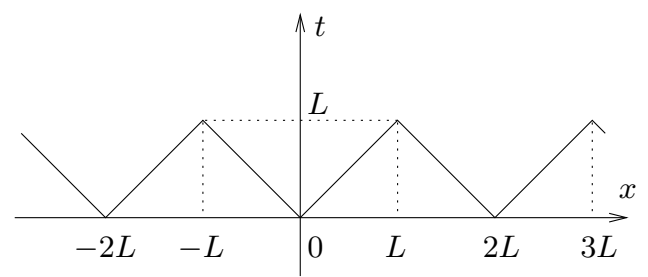

Figure 3: The piecewise-null surface $S_{0}(x)$ having zero proper length.

(see Fig. 31). It is clear that the proper length (i.e. the "1-volume") of this hypersurface is equal to zero. We can then choose a smooth spacelike hypersurface situated sufficiently close to $t=S_{0}(x)$, so that the proper length of the portion of that hypersurface corresponding to $|x|<L$ is smaller than a chosen bound $\varepsilon$. It is also possible to find another hypersurface for $L<|x|<2 L$ approaching the null surface $S_{0}(x)$ even closer, so that the corresponding proper length is smaller than $\varepsilon / 2$, and so on. Thus there exists an unbounded spacelike hypersurface $S_{\varepsilon}(x)$ with a finite and arbitrarily small proper length $\mathcal{L}\left[S_{\varepsilon}\right]<\varepsilon$.

The Minkowski space can be foliated by copies of the surface $t=S_{0}(x)$ shifted in the $t$ direction. Instead of this "null foliation" we consider a family of spacelike hypersurfaces labeled by a time parameter $\tau$,

$$
t=t(\tau, x) \equiv \tau+S_{\varepsilon(\tau)}(x),
$$

where $\varepsilon(\tau)$ is some function. The family (B3) will be an admissible foliation of the 1+1-dimensional Minkowski spacetime as long as $\partial t / \partial \tau>0$ and each hypersurface is spacelike,

$$
\left.\left(d t^{2}-d x^{2}\right)\right|_{t=t(\tau, x), \tau=\mathrm{const}}<0 .
$$

These conditions can be satisfied by a suitable choice of the function $\varepsilon(\tau)$, and moreover it is possible to have $\varepsilon(\tau)$ monotonically decrease to zero as $\tau \rightarrow \infty$. The resulting family of hypersurfaces is the desired foliation of the Minkowski space with a finite and monotonically decreasing proper volume.

This construction can be generalized to the 3+1dimensional Minkowski spacetime by considering a family of spherically symmetric hypersurfaces

$$
t=t(\tau, r)=\tau+S_{\varepsilon(\tau)}(r), \quad r \equiv|\mathbf{x}| .
$$

As will be demonstrated below, the function $\varepsilon(\tau)$ can be chosen to achieve a finite proper volume $\mathcal{V}(\tau)$ of the entire equal- $\tau$ hypersurface and moreover to have $\mathcal{V}(\tau) \rightarrow 0$ as $\tau \rightarrow \infty$.

A slightly modified but basically similar construction yields an analogous family of spacelike hypersurfaces foliating a de Sitter spacetime. For simplicity we consider a 1+1-dimensional de Sitter spacetime with flat spatial sections. Then we can use conformal coordinates $(\eta, x)$, 


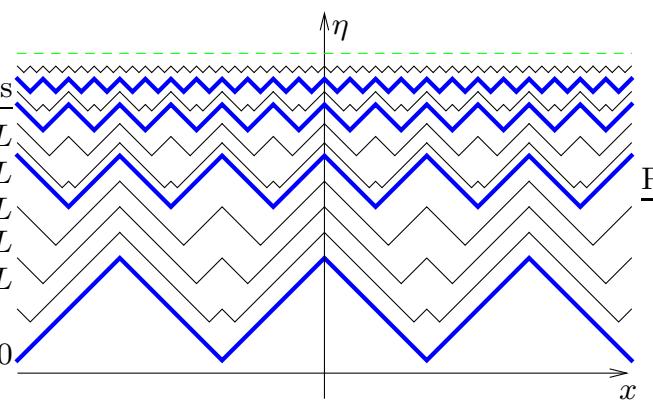

Figure 4: A null foliation of the de Sitter spacetime in conformal coordinates $(\eta, x)$. Thicker lines are the consecutively smaller copies of the saw-shaped surface $S_{0}(x)$, while the thinner lines in between show the continuous deformation procedure ("growing ridges"). The dashed line corresponds to an infinite future $(\eta \rightarrow 0)$.

so that the de Sitter line element is conformally flat,

$$
\begin{aligned}
d s^{2} & =d t^{2}-e^{-2 H t} d x^{2}=\frac{1}{H^{2} \eta^{2}}\left(d \eta^{2}-d x^{2}\right), \\
\eta & =-\frac{1}{H} e^{-H t}, \quad-\infty<\eta<0 .
\end{aligned}
$$

All lines $\eta=\eta_{0} \pm x$ are null geodesics and thus the sawshaped hypersurface $\eta=S_{0}(x)$ is piecewise null. However, we cannot use the hypersurface $\eta=S_{0}(x)$ to foliate the spacetime as in the Minkowski case because the boundary $\eta=0$ prevents us from shifting $S_{0}(x)$ arbitrarily in $\eta$. Instead it is possible to continuously deform the "saw" and to decrease the size of its ridges while growing new ridges as time passes (see Fig. (4). The resulting family of null hypersurfaces $S_{0}(\tau, x)$ can be labeled by a conformal time parameter $\tau$, where $\tau<0, \tau \rightarrow 0$ as $\eta \rightarrow 0$, and it is clear from the figure that $S_{0}(\tau, x)$ is a foliation of the entire future part of the de Sitter spacetime. The piecewise-null hypersurfaces $S_{0}(\tau, x)$ are then used as a "skeleton" for a family of non-intersecting, smooth spacelike hypersurfaces $\eta=H_{\varepsilon}(\tau, x)$ with a finite proper volume $\mathcal{V}\left[H_{\varepsilon}\right]<\varepsilon$. It is possible to choose the function $\varepsilon(\tau)$ such that $\varepsilon(\tau) \rightarrow 0$ as $\tau \rightarrow 0$. This procedure constructs a foliation of the de Sitter spacetime with the total proper volume decreasing to zero at late times.

In the remainder of this appendix I describe the required hypersurfaces explicitly and verify their properties as claimed.

The first step is to replace the angular piecewise-null hypersurface $S_{0}(x)$ by a smooth hypersurface $S_{0}(x ; \varepsilon)$ which is null everywhere except the "caps" at the corners. To this end we use a "smooth corner" function $C(x)$ which is infinitely differentiable, convex, and satisfies $C(x)=|x|$ for $|x| \geq 1$ and $\left|C^{\prime}\right|<1$ for $|x|<1$. An example of a suitable function $C(x)$ is plotted in Fig. [5

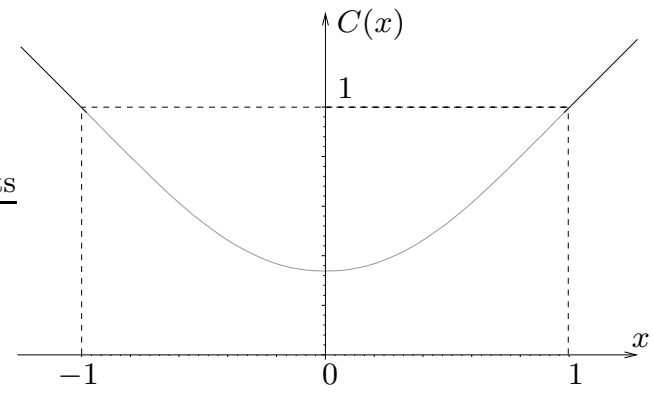

Figure 5: The "smooth corner" function $C(x)$ defined by Eq. (B8) with $A=0.01, B=0.5, C_{1} \approx 0.6613, C_{2} \approx 3.323$.

and is defined for $|x|<1$ by

$C(x) \equiv 1-C_{1}+C_{2} \int_{0}^{|x|} d y \int_{0}^{y} d z \exp \left(-\frac{A}{z}-\frac{B}{1-z}\right)$,

where $A, B$ are positive constants and

$$
\begin{aligned}
C_{1} & \equiv C_{2} \int_{0}^{1} d y \int_{0}^{y} d z \exp \left(-\frac{A}{z}-\frac{B}{1-z}\right), \\
C_{2} & \equiv\left[\int_{0}^{1} d z \exp \left(-\frac{A}{z}-\frac{B}{1-z}\right)\right]^{-1} .
\end{aligned}
$$

The function $C(x)$ is smooth because by construction all derivatives of $C(x)$ vanish at $x=0$ and at $x= \pm 1$ [except $C^{\prime}( \pm 1)= \pm 1$ ], while at other $x$ the function $C(x)$ is analytic. Note also the inequality

$$
0 \leq C(x)-x C^{\prime}(x)<1
$$

which follows from the convexity of $C(x)$.

A rescaled "smoothed corner" function $C(x)$ will be used to replace small neighborhoods of the top and the bottom corners of the curve $S_{0}(x)$. Namely, we define the "rescaled corner" functions

$$
C_{\delta}(x) \equiv C\left(x \delta^{-1}\right) \delta, \quad \tilde{C}_{\delta}(x) \equiv L-C_{\delta}(x),
$$

plotted in Fig. [ A spherically symmetric hypersurface $t=C_{\delta}(|\mathbf{x}|)$ is null except for a small range $|\mathbf{x}|<\delta$, and its proper 3-volume is smaller than the volume of a sphere with radius $\delta$. We now replace the corners at $|x|=n L$ in the curve $S_{0}(x)$ by smoothed corners $C_{\delta_{n}}(x)$ or $\tilde{C}_{\delta_{n}}(x)$, where $\delta_{n}$ decreases with $n$. For later convenience we set

$$
\delta_{0} \equiv \varepsilon L ; \quad \delta_{n} \equiv \varepsilon L n^{-2} 2^{-n}, \quad n=1,2, \ldots,
$$

where $\varepsilon<1$ is a free dimensionless parameter. To be definite, let us choose the smoothed-out version of $S_{0}(x)$ as follows,

$$
\begin{aligned}
& S_{0}(x ; \varepsilon) \equiv \\
& \left\{\begin{array}{l}
C_{\delta_{n}}(x-2 n L), \quad 2 n-\frac{1}{2} \leq \frac{|x|}{L} \leq 2 n+\frac{1}{2} ; \\
\tilde{C}_{\delta_{n}}(x-(2 n+1) L), \quad 2 n+\frac{1}{2} \leq \frac{|x|}{L} \leq 2 n+\frac{3}{2},
\end{array}\right.
\end{aligned}
$$




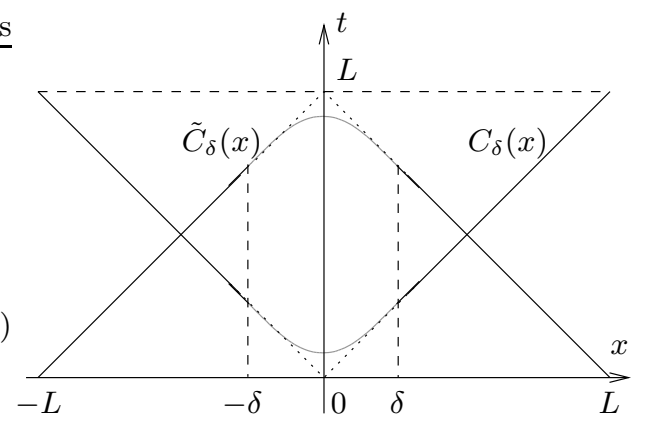

Figure 6: The rescaled "smooth corner" functions $C_{\delta}(x)$ (corner down) and $\tilde{C}_{\delta}(x)$ (corner up) defined by Eq. (B12).

where $n=0,1,2, \ldots$ By construction, the function $S_{0}(x ; \varepsilon)$ has the following properties that will be used below:

$$
\begin{aligned}
& 0 \leq S_{0}(x ; \varepsilon) \leq L, \quad \frac{\partial}{\partial x} S_{0}(x ; \varepsilon) \leq 1 \\
& \left|\frac{\partial}{\partial \varepsilon} S_{0}(x ; \varepsilon)\right| \leq \sup _{n, x}\left|\frac{\partial C_{\delta_{n}}(x)}{\partial \varepsilon}\right|<\varepsilon L \leq L .
\end{aligned}
$$

The last property can be derived from Eq. (B11).

The hypersurface $t=S_{0}(|\mathbf{x}| ; \varepsilon)$ is not everywhere null because of thin spacelike spherical shells in small neighborhoods of $|\mathbf{x}|=n L, n=0,1,2, \ldots$ The 3 -volume of these shells can be estimated as

$$
\begin{aligned}
\mathcal{V}\left[S_{0}(x ; \varepsilon)\right] & <\frac{4 \pi}{3} \sum_{n=0}^{\infty}\left[\left(n L+\delta_{n}\right)^{3}-\left(n L-\delta_{n}\right)^{3}\right] \\
& <\frac{4 \pi}{3}\left(\delta_{0}^{3}+6 L^{2} \sum_{n=1}^{\infty} n^{2} \delta_{n}\right)<30 \varepsilon L^{3} .
\end{aligned}
$$

Therefore the total volume of all the "smoothing caps" can be made negligible by choosing a sufficiently small $\varepsilon$.

Now we are ready to describe the foliation of the $3+1$ dimensional Minkowski spacetime. The spherically symmetric hypersurface $t=S_{\varepsilon}(\tau, r)$ is constructed by rescaling and shifting the smoothed function $S_{0}(x ; \varepsilon)$ defined by Eq. (B14). A particular ansatz can be written as

$$
S_{\varepsilon}(\tau, r)=\tau+(1-h(\tau, r)) S_{0}(r ; \varepsilon(\tau)),
$$

where $h(\tau, r)$ and $\varepsilon(\tau)$ are suitable functions and $0<$ $h(\tau, r)<1$. By construction, the surface $S_{\varepsilon}$ is everywhere smooth (infinitely differentiable) and approaches the "skeleton" surface $S_{0}$ as $h \rightarrow 0$, and we shall set

$$
h(\tau, r)=\varepsilon \exp \left(-\frac{r+\tau}{2 L}\right), \quad \varepsilon(\tau)=\varepsilon \exp \left(-\frac{\tau}{4 L}\right) .
$$

It then follows from Eq. B15 that

$$
\begin{aligned}
0 & \leq S_{\varepsilon}(\tau, r)-\tau \leq(1-h) L<L, \\
\left|\frac{\partial h}{\partial r}\right| & \leq \frac{1}{2 L} h, \\
\left|\frac{\partial}{\partial r} S_{\varepsilon}(\tau, r)\right| & \leq 1-h+L\left|\frac{\partial h}{\partial r}\right| \leq 1-\frac{h}{2}<1 .
\end{aligned}
$$

Therefore the hypersurface $t=S_{\varepsilon}(\tau, r)$ is everywhere spacelike. The proper 3 -volume of this hypersurface is

$$
\mathcal{V}\left[S_{\varepsilon}(\tau, r)\right]=4 \pi \int_{0}^{\infty} d r \sqrt{{ }^{(3)} g(r)}
$$

where ${ }^{(3)} g$ is the determinant of the induced metric within the surface:

$$
\begin{aligned}
d s^{2} & =\left[1-\left(\frac{\partial S_{\varepsilon}}{\partial r}\right)^{2}\right] d r^{2}+r^{2} d \Omega^{2} ; \\
{ }^{(3)} g(r) & =\left[1-\left(\frac{\partial S_{\varepsilon}}{\partial r}\right)^{2}\right] r^{4} .
\end{aligned}
$$

We can now divide the hypersurface into the "caps," i.e. ranges of $r$ within small neighborhoods of $n L, n=$ $0,1,2, \ldots$, and "hills" where $r$ is outside those neighborhoods. For $r$ within the "hills" we have $\partial S_{0} / \partial r= \pm 1$ and thus the following upper bound holds for those $r$,

$$
\begin{aligned}
1-\left(\frac{\partial S_{\varepsilon}}{\partial r}\right)^{2}= & 1-(1-h)^{2}\left(\frac{\partial S_{0}}{\partial r}\right)^{2} \\
& +2 \frac{\partial h}{\partial r} \frac{\partial S_{0}}{\partial r} S_{0}(1-h)-\left(\frac{\partial h}{\partial r}\right)^{2} S_{0}^{2} \\
\leq & 2 h+2\left|\frac{\partial h}{\partial r}\right| S_{0} \leq 2 h+2 \frac{h}{L} L<4 h .
\end{aligned}
$$

So the total volume of the "hills" is less than

$$
4 \pi \int_{0}^{\infty} r^{2} \sqrt{4 h(\tau, r)} d r=2048 \pi \varepsilon L^{3} \exp \left(-\frac{\tau}{4 L}\right) .
$$

The total volume of the "caps" is constrained by Eq. (B17), where $\varepsilon$ is now replaced by the function $\varepsilon(\tau)$ defined by Eq. (B19). Therefore an upper bound on the total volume is found as

$$
\begin{aligned}
\mathcal{V}\left[S_{\varepsilon}(\tau, r)\right] & <30 \varepsilon(\tau) L^{3}+4 \pi \int_{0}^{\infty} r^{2} \sqrt{4 h(\tau, r)} d r \\
& <6464 \varepsilon L^{3} \exp \left(-\frac{\tau}{4 L}\right) .
\end{aligned}
$$

As we intended, the total 3-volume of the hypersurface $t=S_{\varepsilon}(\tau, r)$ is finite and approaches zero at late times $(\tau \rightarrow \infty)$.

Finally we need to check that the hypersurfaces $t=$ $S_{\varepsilon}(\tau, r)$ are strictly monotonic in $\tau$, i.e. $\partial S_{\varepsilon} / \partial \tau>0$. 


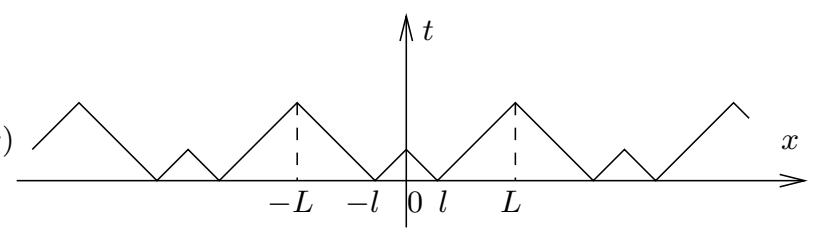

Figure 7: A typical piecewise-null hypersurface from the foliation in Fig. 4

Using Eqs. (B16) and (B19), we find

$$
\begin{aligned}
\left|\frac{\partial h}{\partial \tau}\right| & \leq \frac{1}{2 L} h, \quad 1-\frac{\partial h}{\partial \tau} S_{0} \geq 1-\frac{h}{2}>\frac{1}{2} \\
\frac{\partial S_{\varepsilon}}{\partial \tau} & =1-\frac{\partial h}{\partial \tau} S_{0}+(1-h) \frac{\partial S_{0}(r ; \varepsilon)}{\partial \varepsilon} \frac{d \varepsilon(\tau)}{d \tau} \\
& >\frac{1}{2}-\frac{1}{4 L} L>0 .
\end{aligned}
$$

Therefore the family of hypersurfaces $S_{\varepsilon}(\tau, r)$ is indeed a foliation of the entire Minkowski spacetime. This argument completes the construction.

Turning now to the future part of a de Sitter spacetime, say $-H^{-1}<\eta<0$, we start with the family of piecewise-

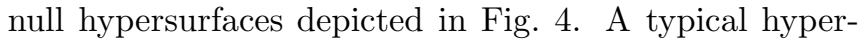
surface having smaller ridges of size $l$ and larger ridges of size $L$ is shown in Fig. 7 We denote by $S_{\varepsilon}(x ; l, L)$ a smoothed version of this hypersurface constructed using the "corner" functions (B12); a cumbersome explicit formula for $S_{\varepsilon}(x ; l, L)$ can be written which we omit. One can easily verify the properties

$$
\left|S_{\varepsilon}(x ; l, L)\right| \leq L,\left|\frac{\partial S_{\varepsilon}}{\partial x}\right| \leq 1, \frac{\partial S_{\varepsilon}}{\partial l} \geq 0,\left|\frac{\partial S_{\varepsilon}}{\partial \varepsilon}\right| \leq L .
$$

The desired family of smooth hypersurfaces $\eta=$ $H_{\varepsilon}(\tau, r)$ is built, as before, by shifting and rescaling the functions $S_{\varepsilon}(x ; l, L)$. An additional time dependence in the parameters $l$ and $L$ is needed to reproduce the be-

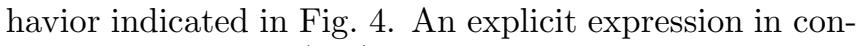
formal coordinates $(\eta, r)$ can be written as

$$
\eta=H_{\varepsilon}(\tau, r)=\tau+(1-h(\tau, r)) S_{\varepsilon(\tau)}(r ; l(\tau), L(\tau)),
$$

where we consider the range $-H^{-1}<\tau<0$ (recall that $\tau$ is a conformal time parameter), the functions $h(\tau, r)$ and $\varepsilon(\tau, r)$ are similar to those in the Minkowski case, and we define

$$
\begin{aligned}
L(\tau) & =2^{-p(\tau)} L, \quad p(\tau) \equiv-\left\lfloor\log _{2}(-H \tau)\right\rfloor, \\
l(\tau) & =2^{-p(\tau)} L+\frac{1}{2} H \tau L,
\end{aligned}
$$

where $\lfloor x\rfloor$ denotes the integer part of $x$, i.e. the algebraically largest integer not exceeding $x$. The definitions (B34)-(B35) correspond to Fig. [4 and describe a gradual growth of ridges with size $l(\tau)$. By construction $L(\tau) \leq L|H \tau|$; more precisely, during the time range
$-H^{-1} 2^{-p+1}<\tau \leq-H^{-1} 2^{-p}$ we have $L(\tau)=L 2^{-p}$ while the ridge size $l(\tau)$ changes from 0 to $L 2^{-p-1}$. At $\tau=-H^{-1} 2^{-p}$ the ridge scale $L$ is halved and $l$ is reset to zero. This requires $p \geq 0$, i.e. $\tau \geq-2 H^{-1}$, and the latter inequality holds since we consider only the range $-H^{-1} \leq \tau<0$.

The 3 -volume of the hypersurface $H_{\varepsilon}(\tau, r)$ is found as

$$
\mathcal{V}\left[H_{\varepsilon}(\tau, r)\right]=\frac{4 \pi}{H^{3}} \int_{0}^{\infty} r^{2} d r \frac{\sqrt{1-\left(\partial H_{\varepsilon} / \partial r\right)^{2}}}{H_{\varepsilon}^{3}} .
$$

Since the entire hypersurface is bounded in time, namely $H_{\varepsilon}(\tau, r) \leq \frac{1}{2} \tau$, we may estimate its 3 -volume from above using the maximum scale factor $8|H \tau|^{3}$. An upper bound on the factor $\sqrt{1-\left(\partial H_{\varepsilon} / \partial r\right)^{2}}$ is obtained similarly to Eq. (B26) since the function $S_{\varepsilon}(r ; l, L)$ has properties (B32) similar to those of $S_{0}(x ; \varepsilon)$, so

$$
1-\left(\frac{\partial H_{\varepsilon}}{\partial r}\right)^{2}<4 h(\tau, r)
$$

We now choose $\varepsilon(\tau)$ and $h(\tau, r)$ as before except for the replacement $\tau \rightarrow-H^{-1} \ln (-H \tau)$ as appropriate for the conformal time,

$\varepsilon(\tau) \equiv \varepsilon \exp \left[\frac{\ln |H \tau|}{4 H L}\right], h(\tau, r) \equiv \varepsilon \exp \left[\frac{\ln |H \tau|}{2 H L}-\frac{r}{2 L}\right]$.

Then the total volume of the hypersurface is estimated similarly to Eq. (B28),

$$
\begin{aligned}
\mathcal{V}\left[H_{\varepsilon}\right] & <8|H \tau|^{-3}\left[30 \varepsilon(\tau) L^{3}+4 \pi \int_{0}^{\infty} r^{2} \sqrt{4 h(r)} d r\right] \\
& <51712 \varepsilon L^{3}|H \tau|^{\frac{1}{4 H L}-3} .
\end{aligned}
$$

Choosing the free parameter $L$ (the ridge size) such that $H L<\frac{1}{12}$, i.e. having more than 12 ridges per Hubble length, we find

$$
\lim _{\tau \rightarrow 0} \mathcal{V}\left[H_{\varepsilon}(\tau, r)\right]=0
$$

as required.

It remains to verify that the hypersurfaces $\eta=H_{\varepsilon}(\tau, r)$ are everywhere spacelike and monotonic in $\tau$. Note that the functions $\varepsilon(\tau)$ and $h(\tau, r)$ satisfy

$$
\left|\frac{d \varepsilon}{d \tau}\right|=\frac{\varepsilon(\tau)}{4 L|H \tau|} \leq \frac{\varepsilon(\tau)}{4 L(\tau)} ;\left|\frac{\partial h}{\partial \tau}\right|=\frac{h(\tau, r)}{2 L|H \tau|} \leq \frac{h}{2 L(\tau)} .
$$

The spacelike character is proved by the estimate

$$
\left|\frac{\partial H_{\varepsilon}}{\partial r}\right|<1
$$

which is derived from Eq. (B32):

$$
\begin{aligned}
\left|\frac{\partial}{\partial r} H_{\varepsilon}(\tau, r)\right| & \leq(1-h)\left|\frac{\partial}{\partial r} S_{\varepsilon}(\tau, r)\right|+\left|\frac{\partial h(\tau, r)}{\partial r}\right| S_{\varepsilon}(\tau, r) \\
\leq & 1-h+\frac{h}{2 L} L(\tau) \leq 1-h+\frac{h}{2}=1-\frac{h}{2}<1 .
\end{aligned}
$$


The monotonicity, $\partial H_{\varepsilon} / \partial \tau>0$, can be shown using Eq. (B32) and the following inequalities,

$$
\begin{aligned}
\frac{\partial H_{\varepsilon}}{\partial \tau} & =1-\frac{\partial h}{\partial \tau} S_{\varepsilon}+(1-h)\left[\frac{\partial S_{\varepsilon}}{\partial \varepsilon} \frac{d \varepsilon(\tau)}{d \tau}+\frac{\partial S_{\varepsilon}}{\partial l} \frac{d l(\tau)}{d \tau}\right] \\
& >1-\frac{h}{2 L(\tau)} L(\tau)-\frac{\varepsilon}{4 L(\tau)} L(\tau) \geq 1-\frac{1}{2}-\frac{1}{4}>0 .
\end{aligned}
$$

[1] A. Vilenkin, Phys. Rev. D 27, 2848 (1983).

[2] A. D. Linde, Phys. Lett. B 175, 395 (1986).

[3] A. Starobinsky, in Current Topics in Field Theory, Quantum Gravity and Strings (eds. H. J. de Vega and N. Sanchez), Lecture Notes in Physics 246, p. 107 (SpringerVerlag, Heidelberg, 1986).

[4] A. D. Linde, D. A. Linde, and A. Mezhlumian, Phys. Rev. D 49, 1783 (1994) hep-th/9408023.

[5] J. Garcia-Bellido and A. D. Linde, Phys. Rev. D 51, 429 (1995) hep-th/9408023.

[6] A. Vilenkin, Phys. Rev. D 52, 3365 (1995) gr-qc/9505031.

[7] A. Vilenkin, Phys. Rev. Lett. 81, 5501 (1998) hep-th/9806185.

[8] V. Vanchurin, A. Vilenkin, and S. Winitzki, Phys. Rev. D 61, 083507 (2000) gr-qc/9905097.

[9] J. Garriga and A. Vilenkin, Phys. Rev. D 64, 023507 (2001) gr-qc/0201090.
This argument completes the construction of the foliation for the de Sitter spacetime.
[10] S. Hawking, "Cosmology from the top down," talk transcript astro-ph/0305562.

[11] S. Gratton and N. Turok, "Langevin analysis of eternal inflation" hep-th/0503063.

[12] B. Mandelbrot, The Fractal Geometry of Nature (Freeman, New York, 1983).

[13] K. B. Athreya and P. E. Ney, Branching Processes (Springer-Verlag, New York, 1972).

[14] A. H. Guth and E. J. Weinberg, Nucl. Phys. B 212, 321 (1983).

[15] A. Vilenkin, Phys. Rev. D 59, $123506 \quad$ (1999) gr-qc/9902007.

[16] S. Winitzki and A. Vilenkin, Phys. Rev. D 53, 4298 (1996) gr-qc/9510054.

[17] S. Winitzki, Phys. Rev. D 65, 083506 (2002) gr-qc/0111048.

[18] M. Aryal and A. Vilenkin, Phys. Lett. B 199, 351 (1987). 